\title{
Heart Retransplantation: Candidacy, Outcomes, and Management
}

\author{
Maya H. Barghash ${ }^{1,2}$ • Sean P. Pinney ${ }^{1,2}$
}

Published online: 17 December 2019

(C) Springer Nature Switzerland AG 2019

\begin{abstract}
Purpose of Review To describe the incidence and epidemiology of heart retransplantation in adults and children and to review the risk factors associated with adverse outcome following retransplantation to help guide recipient selection.

Recent Findings Heart retransplantation is associated with inferior short-term and long-term survival when compared with primary heart transplantation and its use remains controversial although less so in the pediatric heart transplant population.

Summary In the most recent era of heart transplantation, patients retransplanted for CAV, greater than 1 year from their primary transplant, and who are not in critical condition have improved survival compared with other retransplant recipients. Survival with retransplantation can approach that of primary transplantation when patients are appropriately selected. More research is needed regarding the optimal timing for retransplantation and the optimal management for patients after retransplantation.
\end{abstract}

Keywords Heart transplant $\cdot$ Retransplantation $\cdot$ Advanced heart failure

\section{Introduction}

Heart transplantation is the gold standard treatment for stage $\mathrm{D}$ heart failure with 35,703 adult heart transplants performed worldwide between 2009 and 2017 and approximately 5800 performed annually [1]. Median survival after adult heart transplantation exceeds 12 years, survival conditional on 1year survival is greater than $50 \%$ at 14 years of follow-up [1], and unadjusted 1-year survival after heart transplantation in the most recent era is $85 \%$ [1,2 $\bullet$. Complications after transplant include graft failure, rejection, and cardiac allograft vasculopathy (CAV). Cardiac retransplantation is the only durable long-term treatment option when end-stage allograft failure occurs as a result of these complications [1].

The number of cardiac retransplants performed has remained steady over time between 100 and 120 annually worldwide or between 2 and $4 \%$ of all adult heart transplants. Due to the increasing number of heart transplants in the recent

This article is part of the Topical Collection on Thoracic Transplantation

Maya H. Barghash

Maya.barghash@mountsinai.org

1 Zena and Michael A. Wiener Cardiovascular Institute, Icahn School of Medicine at Mount Sinai, New York, USA

2 The Mount Sinai Hospital, 1190 Fifth Avenue, Box 1030, New York, NY 10029, USA era, retransplantation accounts for a smaller proportion of all heart transplantations performed (2.2\% in 2016) [1]. In light of the donor shortage and inferior outcomes seen with retransplantation as compared with primary transplantation, gaining an understanding of factors that affect outcome with retransplantation is paramount to being proper stewards of this precious resource and selecting patients that are most likely to benefit from this therapy.

\section{Cardiac Retransplantation}

\section{Incidence and Epidemiology of Heart Retransplantation}

The rates of retransplantation have been relatively steady over the years accounting for 2-4\% of all adult heart transplants. Approximately $40 \%$ of retransplantations occur 10 or more years from the index heart transplant, reflecting the median survival seen with transplant [2•]. Retransplantation accounts for approximately $5 \%$ of heart transplants performed in the younger age group between 18 and $39[2 \cdot]$.

Compared with primary heart transplant recipients, retransplant recipients are on average younger, more sensitized, and tend to be more acutely ill with worse renal function, increased likelihood of being hospitalized, on dialysis, intubated, on inotropes, or supported with extracorporeal 
membrane oxygenation (ECMO) [2•]. In the most recent era between 2006 and 2013, 51.6\% of adult heart retransplant recipients were hospitalized at the time of retransplant, $48 \%$ were on intravenous inotropes, $6.7 \%$ bridged with an LVAD, $4.6 \%$ bridged with an RVAD, $7.2 \%$ with an IABP, $8 \%$ with a ventilator, $2 \%$ with a total artificial heart (TAH), and $5.8 \%$ with ECMO [2•]. Adult retransplant recipients are more sensitized than those undergoing primary heart transplantation, with $43 \%$ of retransplant recipients with a PRA of $10 \%$ or higher compared with $18.3 \%$ of primary adult heart transplant recipients $[2 \bullet]$.

The most common indications for retransplantation are CAV and allograft failure. While primary graft failure is the most common reason in the first month after heart transplantation, CAV is the most common cause after the first year. Other reasons include transplant rejection or valvular disease [3]. An earlier analysis of the ISHLT/UNOS registry for all cardiac retransplantations performed in the USA from 1987 to 1998 described time from the primary transplant to retransplantation to range from 1 day to 15.5 years, with $56 \%$ undergoing retransplantation for chronic rejection or CAV, $18 \%$ for primary or nonspecific graft failure, $9 \%$ for acute rejection, and $3 \%$ due to hyperacute rejection. The majority of these patients $(60 \%)$ were in the intensive care unit (ICU) at the time of retransplantation and $40 \%$ were supported with some form of life support (i.e., ventricular assist device (VAD), ventilator, inotropes) [3].

The presence of severe CAV (left main stenosis $\geq 50 \%$, or two or more primary vessels stenoses $\geq 70 \%$, or branch stenosis $\geq 70 \%$ in all 3 systems) is associated with poor 1 -year survival with high rates of death and retransplantation should be considered in these patients $[4,5]$.

\section{Outcomes with Heart Retransplantation}

In general, short- and long-term survival is lower in heart retransplant recipients compared with first-time heart transplantation [1]. Not only is 1-year survival significantly lower, but survival conditional on 1-year survival is also lower with retransplantation [2•]. When examining ISHLT data for all heart transplantations performed between 1982 and 2016, 1year survival for retransplantation is $68.9 \%$ with a median survival of 6.6 years, median survival conditional on 1-year survival of 11.6 years, and 10-year survival of $38 \%$ [1, 2•]. Survival with retransplantation has improved in the most recent era between 2009 and 2016, with 1-year survival for retransplantation of $80 \%$ compared with $85.4 \%$ 1-year survival for primary adult heart transplants, and 5-year survival of $68.6 \%$ vs. $74.6 \%$ [1]. Similar outcomes have been found when analyzing the UNOS database between 1995 and 2012, with estimated survival at $1,3,5,10$, and 15 years following retransplantation of $80 \%, 70 \%, 64 \%, 47 \%$, and $30 \%$ respectively [6].

Various donor and recipient factors play a role in the inferior outcomes seen with retransplantation. An analysis of the Scientific Registry of Transplant Recipients (SRTR) in the USA between 2000 and 2005 observed 364 heart retransplants performed accounting for $2.9 \%$ of all adult heart transplants. Similar to ISHLT registry data, 1-year, 3-year, and 5-year unadjusted graft survival was lower in retransplants compared with first transplants ( $82 \%$ vs. $86 \%, 70 \%$ vs. $80 \%$, and $58 \%$ vs. $73 \%$ respectively, $p<0.0001)$. However, when adjusting for donor and recipient factors, the relative risk for graft loss with retransplantation was not significantly different from first heart transplantation. Recipient factors adjusted for included age, diagnosis, medical condition, creatinine, ventilator status, and use of extracorporeal membrane oxygenator (ECMO) suggesting that retransplant recipients tend to be more critically ill with more comorbidities. Donor factors adjusted for included age, cause of death, and cold ischemic time [7, 8]

There are inherent challenges to risk stratification of recipients of cardiac retransplantation but it remains an important topic of discussion given the overall shortage of donor organs and reduced survival compared with first-time transplant. Risk factors for increased mortality after retransplant include short inter-transplantation interval; transplant center volume; use of a ventricular assist device (VAD), ECMO, or ventilator; ICU status; and older recipient age [3]. Similar risk factors were identified in an analysis of the UNOS registry as well as long ischemic time [9]. The ISHLT registry did not identify recipient age as a significant predictor for survival after retransplantation [2•] but did find that younger donor age (11 to 39 years) was associated with improved median survival in retransplantation [2•]. Retransplantation within the first year after primary heart transplant is associated with lower 1year survival compared with those who are retransplanted with a longer inter-transplant interval. The ISHLT registry data evaluating the cohort of heart retransplants between 2009 and 2016 found an inter-transplant interval of 1 year or less to be significantly associated with lower 1-year survival of $57.9 \%$ vs. $85 \%$ if the inter-transplant interval was greater than 1 year [1]. An analysis of the UNOS database from 1987 to 2011 identified 1,690 cardiac retransplants performed with $8.8 \%$ ( $n$ $=149$ ) bridged with mechanical support, and of these 90 (60\%) were supported by VAD, $54(36 \%)$ by ECMO, and 5 (4\%) by ECMO and VAD [10•]. While survival was worse in this cohort compared with those not requiring mechanical support (1-year survival $48 \%$ in those bridged with MCS vs. $71 \%$ in those retransplanted without MCS), those bridged with ECMO had significantly worse survival than those bridged with VAD (1-year survival $40 \%$ with ECMO bridge vs. $53 \%$ with VAD bridge to retransplantation). The median survival was also significantly less with ECMO-bridged retransplants: 7.2 years in retransplants without MCS, 4.3 
years in retransplants bridged with VAD, and 2 months in retransplants bridged with ECMO [10॰]. Notably, the majority of retransplants performed with MCS bridge (68\%) were for graft failure within 1 year of initial heart transplant and thus reflect a sicker patient population. While ECMO use was associated with poor outcomes regardless of time from transplant and era of transplantation, those retransplants performed more than 1 year post-transplant with a VAD bridge had similar survival to retransplants performed without MCS bridge, and survival was also improved if the retransplant was performed between 2003 and 2011 (1-year survival 67\%) vs. 1987 and 2002 (1-year survival 37\%).

Other factors affecting outcomes with retransplantation are related to the indication for retransplantation. Retransplantation for CAV has the best prognosis owing to greater 1-year survival of $74.7 \%$ [2•] (Fig. 1). Single-center experiences in large-volume centers have also shown favorable outcomes with retransplantation when the cohort of recipients was less critically ill prior to retransplantation and the majority were transplanted for CAV [11]. Between 2006 and 2013, 820 adult heart retransplants were performed in the ISHLT registry: 391 (47.8\%) were performed for CAV, 47 $(5.8 \%)$ for primary graft failure, and $132(16.2 \%)$ for rejection [2•]. Those retransplanted for primary graft failure have poor 1 -year survival of 54\%; however, if they survive the first year, prognosis is comparable with other indications for retransplantation [2•]. In addition to retransplantation for primary graft failure, another poor prognostic indicator that has been identified in other cohorts includes retransplantation for acute rejection, which likely reflects cardiac transplantation performed under urgent circumstances in critically ill immunosuppressed patients [12] as well as higher immune activation placing the retransplanted heart at increased risk of rejection. From the ISHLT registry, 1-year survival for those retransplanted for nonspecific graft failure is $62.7 \%$ and $66.9 \%$ for rejection [2•]. Survival conditional on 1-year survival is similar for all indications for retransplantation [2•].

Renal insufficiency in heart transplant patients is exceedingly common with the majority having some degree of renal insufficiency by 10 years post-transplant. Concurrent kidney transplantation at the time of heart retransplantation versus heart retransplantation alone in patients in need of a second heart transplant is associated with improved survival at 1,5 , and 10 years despite older recipient age and baseline renal function. One-year survival in combined heart retransplant and kidney transplant was $83 \%$ versus $75 \%$ in heart retransplant alone, 5 -year survival of $73 \%$ vs. $56 \%$, and 10 year survival of $50 \%$ vs. $38 \%$ [13]. Of the 820 adult heart retransplantations performed between 2006 and 2012 in the ISHLT registry, heart-kidney transplantation was performed in $91(11 \%)[2 \cdot]$.

\section{Management After Heart Retransplantation}

Rates of hospitalization in the first year following heart retransplantation are high and similar to primary heart transplant recipients, with $46 \%$ admitted in the first year [3]. The most common causes of admission are infection and/or rejection. Early after retransplantation, the leading causes of death are graft failure, multi-organ failure, infection, and acute rejection. Later post-transplant causes include graft failure,

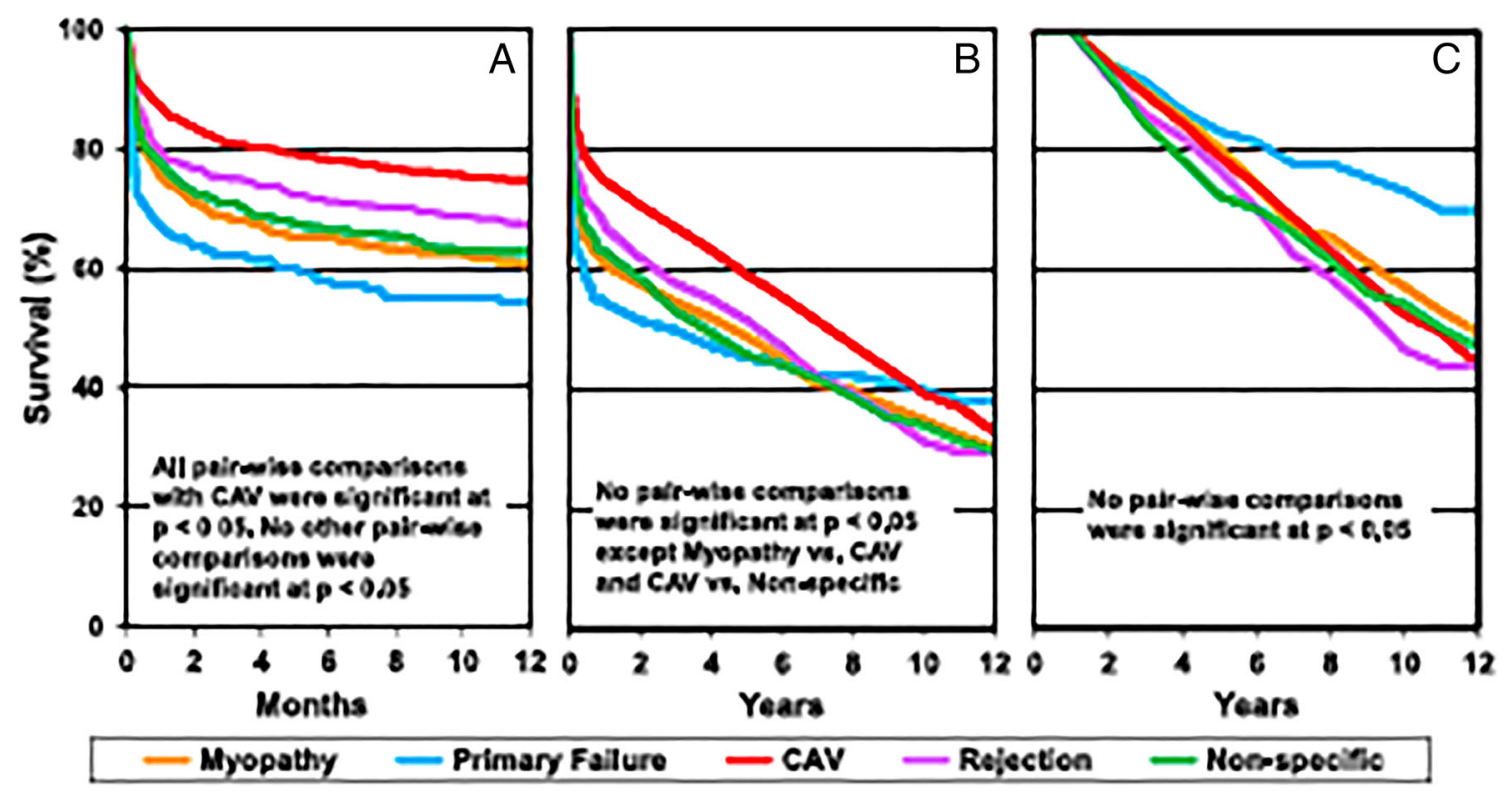

Fig. 1 Survival in adult heart retransplantation stratified by indication. a Kaplan-Meier 1-year survival, b long-term survival, and c long-term survival conditional on survival to 1 year by diagnosis (indication for retransplantation) for adult heart retransplants (1982-June 2012). CAV, cardiac allograft vasculopathy. Used with permission from the International Society for Heart and Lung Transplantation [2•] 
$\mathrm{CAV}$, multi-organ failure, renal failure, infection, and malignancy. In one registry analysis, two-thirds of deaths occurred in either the first 30 days or first year after retransplantation, with a third occurring after the first year [3]. The incidence of any or treated rejection is not significantly different between primary heart transplant and retransplants, and is approximately 25 and $27 \%$ in the first year, respectively [2•].

While single-center studies have found higher rates of malignancy in retransplant recipients [14], similar rates have been observed in the ISHLT registry with an incidence of malignancy of $14.2 \% 5$ years post-primary transplant and $14.7 \% 5$ years post-retransplant, including solid organ malignancy, skin cancer, and lymphoma. With regard to retransplant recipients who had post-transplant lymphoproliferative disorder (PTLD) with their first heart transplant, there is limited data regarding the risk of recurrent PTLD after retransplantation and it remains controversial given the risk of recurrent PTLD with reintroduction of higher levels of immunosuppression. This question was examined in an analysis of the Organ Procurement and Transplant Network (OPTN)/ UNOS database evaluating the safety and efficacy of retransplanting specifically this cohort of patients [15]. Between 1997 and 2005, 69 solid organ transplant patients with a history of PTLD underwent retransplantation, and of these, only 6 were heart transplant patients. The majority (88\%) developed PTLD within 3 years after the first transplant and the indications for retransplantation in this cohort were commonly acute or chronic rejection. Most waited more than 1 year from PTLD diagnosis to retransplantation. The majority of the entire solid organ retransplant cohort $(85.6 \%)$ was alive after 2 years of follow-up with retransplantation with no reports of recurrent PTLD. However, this cohort was compromised predominantly of abdominal organ transplants (kidney and liver) and there was higher mortality seen after thoracic organ retransplantation. When looking at the cases of heart retransplantation, all six were pediatric patients with the majority under the age of 6 years of age. Most were retransplanted within 5 to 10 years of the initial transplant and two of the six died of cardiac causes post-retransplant at 2.5 years of follow-up [14]. Timing of retransplantation in relation to PTLD diagnosis and treatment is an important consideration and a waiting period of 2 years of remission prior to listing has been reported in case reports of favorable outcomes with retransplantation in the kidney transplant literature [16].

There is no significant difference in the use of induction immunosuppression between primary transplant and retransplantation [2•]. Among retransplant recipients, there is no difference in survival based on the use of induction immunosuppression out to 10 years [2•]. Similar to primary transplantation, the majority of retransplant recipients are on a combination of tacrolimus, mycophenolate mofetil, and prednisone at 1 year post-transplant. A quarter of retransplant recipients are on a combination of an mTOR inhibitor as part of maintenance immunosuppression at 5 years post-transplant as compared with $18.7 \%$ of primary transplant recipients; however, this was not compared statistically [2•].

A working group on heart retransplantation summarized indications for retransplantation based on all of the available evidence [7] (Table 1).

\section{Pediatric Heart Retransplantation}

There are unique considerations with regard to heart retransplantation in the pediatric population given the young age at transplant and the short life expectancy of a transplanted heart relative to the age of the child. As such, heart retransplantation in the pediatric population is more often expected when compared with adults.

Pediatric retransplantation accounts for approximately $5 \%$ of all pediatric heart transplants and third and fourth retransplants account for an even smaller portion of this cohort $(<0.5 \%)$. Analysis of pediatric heart transplantations in the ISHLT registry and OPTN database describes a median age of 14 for retransplantation in the pediatric population (range of 1 to 26 years) with a mean inter-transplant interval of $6.8 \pm 5.1$ years after primary transplant $[17,18]$. Indications for retransplantation are similar to adults, with the majority being due to CAV and a smaller percentage due to primary or nonspecific graft failure, acute rejection, chronic rejection, or hyperacute rejection $[17,19]$. Adverse prognostic indicators include short inter-transplant interval of 6 months or less and transplantation for primary graft failure. If this group is excluded, survival with pediatric retransplant was not significantly different than primary pediatric transplantation [18].

Table 1 Indications for retransplantation from the working group on heart retransplantation

1) Chronic severe CAV not amenable to medical or surgical therapy with:

a. Symptoms of ischemia or heart failure and/or

b. Asymptomatic moderate to severe LV dysfunction

2) Chronic graft dysfunction with progressive heart failure in the absence of active rejection with the following considerations:

a. Patients with graft failure due to ongoing acute rejection with hemodynamic compromise, especially less than 6 months post-transplant, are inappropriate retransplant candidates

b. Patients requiring short-term mechanical cardiorespiratory support may not be good retransplant candidates and deserve careful consideration on an individual basis

c. The efficacy of retransplantation in older candidates (age 60-65) is not well established

d. The efficacy of retransplantation in the presence of post-transplant lymphoproliferative disorder (disease-free less than 2 years) is not established

e. Guidelines established for primary transplant candidates should be strictly followed in selecting candidates for retransplantation

From reference [6] 
Other adverse prognostic indicators include the need for MCS, ventilator support, or ECMO; earlier era of transplant; and age older than 18 at the time of retransplant listing [18]. Pediatric retransplant patients are more likely to experience hypertension, hyperlipidemia, renal dysfunction, and are at increased risk for early and late rejection, graft failure, and CAV compared with first time pediatric heart transplantation [17]. In the ISHLT analysis, while early survival rates were similar between primary pediatric heart transplant and retransplantation (1-year survival $84 \%$ in primary pediatric transplantation vs. $81 \%$ in retransplantation; 5 -year survival $72 \%$ vs. $63 \%$ ), late mortality risk was higher with retransplantation (10-year survival $60 \%$ vs. $46 \%, 20$-year survival $42 \%$ vs. $26 \%$ ) with a median survival of 15 years in primary pediatric transplantation vs. 7.3 years in retransplantation [17]. Rates of malignancy are comparable between primary pediatric transplantation and retransplantation.

\section{Conclusions}

Balancing the allocation of a scarce resource and our responsibility to society with our responsibility to individual patients who are in need of a second heart transplant will always be a dilemma for the heart transplant community at large. Every patient must be evaluated on a case by case basis and prior data regarding which patients fare better with heart retransplantation must be taken into account when considering this therapy.

Heart retransplantation in the most recent era of 2002 to 2012 has been associated with improved outcomes. Patients retransplanted for CAV who are not in critical condition requiring an ICU, ventilator, or ECMO, and who are retransplanted more than 1 year after primary transplant, can have outcomes approaching primary heart transplant; however, at best, they remain inferior. While primary graft failure has the worst prognosis of all indications for retransplantation, survival conditional on 1-year survival levels out for all retransplant patients. Other modifiable risk factors may include the use of younger donors (age $\leq 39$ ) and avoiding a long ischemic time. While the use of mechanical circulatory support as a bridge to retransplantation has been associated with lower survival compared with retransplantation without MCS bridge, it is important to note that the majority of these patients studied were bridged with MCS due to early graft failure within 1 year post-primary transplant. When evaluating those bridged with MCS more than 1 year post-transplant in the more recent era, outcomes were similar to those bridged without MCS. Further study is needed in this cohort of patients.

Rates of infection, rejection, and malignancy are similar between primary and retransplant recipients; however, shortand long-term survival is still inferior to primary transplantation and further study is needed to understand not only when to offer retransplantation but how to best manage these candidates after retransplantation.

\section{Compliance with Ethical Standards}

Conflict of Interest Maya Barghash declares no conflict of interest. Sean Pinney reports personal fees from Abbott Inc, Alnylam Inc., Medtronic Inc., and Procyrion Inc., outside the submitted work.

Human and Animal Rights and Informed Consent This article does not contain any studies with human or animal subjects performed by any of the authors.

\section{References}

Papers of particular interest, published recently, have been highlighted as:

- Of importance

1. Khush KK, et al. for the International Society for Heart and Lung Transplantation. The International Thoracic Organ Transplant Registry of the International Society for Heart and Lung Transplantation (ISHLT): thirty-fifth Adult Heart and Lung Transplantation Report - 2018; Focus Theme: Multiorgan Transplantation. JHLT. 2018;30(10):1155-68.

2. Lund LH et al. for the International Society of Heart and Lung Transplantation. The registry of the International Society for Heart and Lung Transplantation: thirty-first official adult heart transplant report - 2014; focus theme: Retransplantation. JHLT 2014; 33(10): 996-1008. This ISHLT report from the international registry focused on providing data on adult heart retransplantation.

3. Srivastava R, Keck BM, Bennett LE, Hosenpud JD. The results of cardiac retransplantation: an analysis of the joint international society for heart and lung transplantation / united network for organ sharing (UNOS) thoracic registry. Transplantation. 2000, 70(4): 606-12.

4. Costanzo MR, Naftel DC, Pritzker MR, Heilman JK, Boehmer JP, Brozena SC, et al. Heart transplant coronary artery disease detected by coronary angiography: a multiinstitutional study of preoperative donor and recipient risk factors. Cardiac transplant research database. J Heart Lung Transplant. 1998;17:744-75.

5. Mehra MR, Crespo-Leiro MG, Dipchand A, Ensminger SM, Hiemann NE, Kobashigawa JA, et al. International Society for Heart and Lung Transplantation working formulation of a standardized nomenclature for cardiac allograft vasculopathy -2010 . J Heart Lung Transplant. 2010;29:717-27.

6. Belli E, Moreno JCL, Hosenpud J, Rawal B, Landolfo K. Preoperative risk factors predict survival following cardiac retransplantation: analysis of the United Network for Organ Sharing database. J Thorac Cardiovasc Surg. 2014;147:1972-7.

7. Johnson MR, Aaronson KD, Canter CE, Kirklin JK, Mancini DM, Mehra MR, et al. Heart retransplantation. Am J Transplant. 2007;7: 2075-81.

8. Magee JC, Barr ML, Basadonna GP, Johnson MR, Mahadevan S, McBride MA, et al. Repeat organ transplantation in the United States, 1996-2005. Am J Transplant. 2007;7(2):1424-33.

9. Shuhaiber JH, Kim JB, Hur K, Gibbons RD, Nemeh HW, Schwartz $\mathrm{JP}$, et al. Comparison of survival in primary and repeat heart 
transplantation from 1987 through 2004 in the United States. Ann Thorac Surg. 2007;83:2135-41.

10. Khan MS, Mery CM, Zafar F, Adachi I, Heinle JS, Cabrera AG, et al. Is mechanically bridging patients with a failing cardiac graft to retransplantation an effective therapy? Analysis of the United Network of Organ Sharing database. J Heart Lung Transplant. 2012;31:1192-8 Only study focusing on mechanically bridged heart retransplant recipients and found that mechanically bridged retransplant patients performed more than one year after index heart transplant had similar outcomes to primary heart transplant.

11. Alturi P, Hiesinger W, Gorman RC, Pochettino A, Jessup M, Acker MA, et al. Cardiac retransplantation is an efficacious therapy for primary cardiac allograft failure. J Cardiothorac Surg. 2008;3(26): $1-5$.

12. Radovancevic B, McGiffin DC, Kobashigawa KA, Cintron GB, Mullen GM, Pitts DE, et al. Naftel DC, and the Cardiac Transplant Research Database Group at the University of Alabama at Birmingham. J Heart Lung Transplant. 2003;22:862-8.

13. Savla J, Lin KY, Pradhan M, Ruebner RL, Rogers RS, Haskins SS, et al. Heart retransplant recipients have better survival with concurrent kidney transplant than with heart retransplant alone. J Am Heart Assoc. 2015;4:e02435. https://doi.org/10.1161/JAHA.115. 002435 .
14. Tsao L, Uriel N, Leitz K, Naka Y, Mancini D. Higher rate of comborbidities after cardiac retransplantation contributes to decreased survival. J Heart Lung Transplant. 2009;28(10):1072-4.

15. Johnson SR, Cherikh WS, Kauffman HM, Pavlakis M, Hanto DW. Retransplantation after post-transplant lymphoproliferative disorders: an OPTN/UNOS database analysis. Am J Transplant. 2006;6:2743-9.

16. Karras A, Thervet E, Le Meur Y, Baudet-Bonneville V, Kessler M, Legendre C. Successful renal retransplantation after post-transplant lymphoproliferative disease. Am J Transplant. 2004;4:1904-9.

17. Conway J, Manlhiot C, Kirk R, Edwards LB, McCrindle BW, Dipchand AI. Mortality and morbidity after retransplantation after primary heart transplant in childhood: an analysis from the registry of the International Society for Heart and Lung Transplantation. J Heart Lung Transplant. 2014;33:241-51.

18. Bock MJ, Nguyen K, Malerba S, Harrison K, Bagiella E, Gelb BD, et al. Pediatric cardiac retransplantation: waitlist mortality stratified by age and era. J Heart Lung Transplant. 2015;34:530-7.

19. Mahle WT, Vincent RN, Kanter KR. Cardiac retransplantation in childhood: analysis of data from the United Network for Organ Sharing. J Thorac Cardiovasc Surg. 2005;130:542-6.

Publisher's Note Springer Nature remains neutral with regard to jurisdictional claims in published maps and institutional affiliations. 